https://doi.org/10.1007/s00350-020-5564-5

\section{Erfolglose Eilanträge im Zusammenhang mit der Corona-Pandemie}

BVerfGG §§32 Abs. 1 i. V. mit 93d Abs. 2

Der Antrag auf Erlass einer einstweiligen Anordnung gegen die bayerische Verordnung über Ausgangsbeschränkungen anlässlich der Corona-Pandemie ist angesichts der überragenden Bedeutung von Leib und Leben abzulehnen - Folgenabwägung

BVerfG, Beschl.v. 7. 4.2020-1 BvR 755/20

Problemstellung: Kurze Zeit nach Inkrafttreten der Verordnungen der Bundesländer über eine vorläufige Ausgangsbeschränkung oder Kontaktverbote anlässlich der COVID-19-Pandemie (CoronaV) erreichten mehrere Eilverfahren auch das BVerfG. Während bislang alle Antragsteller an der Subsidiarität oder an formalen Hindernissen scheiterten (vgl. Az. 1 BvR 661/20; 1 BvR 712/20; 1 BvR 714/20; 1 BvR 742/20; 2 BvR 474/20; 2 BvR 483/20; 2 BvR 571/20), nahm der vorliegende Eilantrag gegen die bay. Ausgangsbeschränkung als erster diese Hürden in der Zulässigkeit. Das BVerfG erachtete es als ,offensichtlich sinn- und aussichtslos“, zunächst bei den Fachgerichten Rechtsschutz zu erlangen, weil diese bereits in anderen Verfahren den Erlass einstweiliger Anordnungen abgelehnt hätten (BayVerfGH, Entsch. v. 26.3.2020 - Vf. 6-VII-20; BayVGH, Beschl. v. 30.3.2020 - 20 NE 20.632). So erging die erste inhaltlich begründete Entscheidung.

Im Eilverfahren hatte die 3. Kammer des Ersten Senats im Rahmen einer Folgenabwägung zu entscheiden, wobei die Auswirkungen auf alle von den angegriffenen Regelungen Betroffenen zu berücksichtigen waren (Rdnr. 8).

Das BVerfG lehnt den Antrag als unbegründet ab. Es betont, dass zwar die Nachteile, die sich aus einer vorläufigen Anwendung ergeben, wenn sich die angegriffenen Maßnahmen im Nachhinein als verfassungswidrig erwiesen, von besonderem Gewicht sind. Sie überwiegen aber nicht deutlich die Nachteile, die entstehen würden, wenn die Maßnahmen außer Kraft träten, sich aber später doch als verfassungsgemäß erweisen würden. Die Gefahren für Leib und Leben wiegen hier schwerer als die Einschränkungen der persönlichen Freiheit. Der Senat erkennt zwar den erheblichen Eingriff in die Grundrechte der Menschen, wenn der Kontakt zueinander eingeschränkt wird, Einrichtungen geschlossen werden und die eigene Wohnung nur noch mit einem bestimmten Grund verlassen werden darf. Ergeht die beantragte einstweilige Anordnung nicht und hätte die Verfassungsbeschwerde Erfolg, sind all diese Einschränkungen mit ihren erheblichen und voraussichtlich teilweise auch unumkehrbaren sozialen, kulturellen und wirtschaftlichen Folgen zu Unrecht verfügt.

Erginge demgegenüber die einstweilige Anordnung und hätte die Verfassungsbeschwerde keinen Erfolg, würden sich nach Einschätzung des Senats voraussichtlich sehr viele Menschen so verhalten, wie es mit den angegriffenen Regelungen unterbunden werden soll, obwohl diese Verhaltensbeschränkungen mit der Verfassung vereinbar wären. Die Richter befürchten, dass dann Einrichtungen, deren wirtschaftliche Existenz

Prof. Dr. iur. Christian Katzenmeier,

Institut für Medizinrecht, Universität zu Köln,

Albertus-Magnus-Platz, 50923 Köln, Deutschland durch die Schließungen beeinträchtigt wird, wieder öffnen, Menschen ihre Wohnung häufig verlassen und auch der unmittelbare Kontakt zwischen Menschen häufig stattfindet. Damit würde sich auch die Gefahr der Ansteckung mit dem Virus, der Erkrankung vieler Personen, der Überlastung der gesundheitlichen Einrichtungen bei der Behandlung schwerwiegender Fälle und schlimmstenfalls des Todes von Menschen nach derzeitigen Erkenntnissen erheblich erhöhen.

Deswegen qualifiziert die Kammer die Folgen einer Fortgeltung der Schutzmaßnahmen bis zur Entscheidung über die Verfassungsbeschwerde zwar als schwerwiegend, aber nicht als unzumutbar. Sie seien vorübergehend hinzunehmen, um einen möglichst weitgehenden Schutz von Gesundheit und Leben zu ermöglichen, zu dem der Staat grundsätzlich auch nach der Verfassung verpflichtet sei. Für die Richter spielt bei der Abwägung auch eine Rolle, dass die Regelungen befristet sind, bezüglich der Ausgangsbeschränkungen viele Ausnahmen vorgesehen werden und bei der Ahndung von Verstößen im Einzelfall im Rahmen des Ermessens individuellen Belangen von besonderem Gewicht Rechnung zu tragen ist (Rdnr. 11).

Über die Verfassungsbeschwerde entscheidet das Gericht zu einem späteren Zeitpunkt. Die Kammer betont: diese „,bedarf eingehenderer Prüfung“ (s. dazu den Besprechungsaufsatz „Grundrechte in Zeiten von Corona“ in diesem Heft).

\section{Christian Katzenmeier}

Aus den Gründen: [1] Die Voraussetzungen zum Erlass der begehrten einstweiligen Anordnung liegen nicht vor.

[2] I. Der Antrag auf Erlass einer einstweiligen Anordnung ist zulässig. Der Bf. ist insbesondere nicht darauf verwiesen, zunächst verwaltungsgerichtlichen Eilrechtsschutz in Anspruch zu nehmen.

[3] Zwar gilt auch im verfassungsgerichtlichen Eilrechtsschutzverfahren der Grundsatz der Subsidiarität der Verfassungsbeschwerde (vgl. \$90 Abs. 2 BVerfGG). Der Erlass einer einstweiligen Anordnung nach $\$ 32$ Abs. 1 BVerfGG kommt daher nur in Betracht, wenn der Bf. beziehungsweise Ast. bestehende Möglichkeiten, fachgerichtlichen Eilrechtsschutz zu erlangen, ausgeschöpft hat (vgl. BVerfG, Beschl. v. 13.8.2019 - 1 BvQ 66/19 -, Rdnr. 2; Beschl. v. 4.12.2019 - 2 BvQ 91/19 -, Rdnr. 2; st. Rspr.).

[4] Dies muss sich der Bf. hier aber nicht entgegenhalten lassen. Neben dem BayVerfGH (Entscheidung v. 26.3.2020 - 6-VII-20 -) hat der BayVerwGH mit Beschl. v. 30.3.2020 - 20 NE 20.632 - im Eilverfahren entschieden, dass die auch hier streitgegenständliche Verordnung des Bayerischen Staatsministeriums für Gesundheit und Pflege vom 24.3.2020 über eine vorläufige Ausgangsbeschränkung anlässlich der Corona-Pandemie nicht außer Vollzug gesetzt wird. Danach erscheint es im Rahmen des Eilrechtsschutzes gegenwärtig unzumutbar, dem Bf. abzuverlangen, zwar nun in eigener Sache, aber zu identischen Rechtsfragen um Eilrechtsschutz nachzusuchen. Jedenfalls insoweit wäre in der zur Verfügung stehenden Zeit die vorherige Anrufung der Fachgerichte derzeit offensichtlich sinn- und aussichtslos (vgl. BVerfGE 55, 154, 157; 70, 180, 186; 145, 20, 54, Rdnr. 85; st. Rspr.).

[5] II. Der Antrag auf Erlass einer einstweiligen Anordnung ist jedoch unbegründet.

[6] 1. Nach $₫ 32$ Abs. 1 BVerfGG kann das BVerfG im Streitfall einen Zustand durch einstweilige Anordnung vorläufig regeln, wenn dies zur Abwehr schwerer Nachteile, zur Verhinderung drohender Gewalt oder aus einem 
anderen wichtigen Grund zum gemeinen Wohl dringend geboten ist. Dabei haben die Gründe, die für die Verfassungswidrigkeit des angegriffenen Hoheitsakts vorgetragen werden, grundsätzlich außer Betracht zu bleiben, es sei denn, die Verfassungsbeschwerde erwiese sich von vornherein als unzulässig oder offensichtlich unbegründet (vgl. BVerfGE 112, 284, 291; 121, 1, 14 f.; st. Rspr.). Bei offenem Ausgang der Verfassungsbeschwerde sind die Folgen, die eintreten würden, wenn die einstweilige Anordnung nicht erginge, die Verfassungsbeschwerde aber später Erfolg hätte, gegenüber den Nachteilen abzuwägen, die entstünden, wenn die begehrte einstweilige Anordnung erlassen würde, der Verfassungsbeschwerde jedoch der Erfolg versagt bliebe (vgl. BVerfGE 131, 47, 55; 132, 195, 232; BVerfG, Beschl. v. 3. 10.2020 - 1 BvQ 15/20 -, Rdnr. 16; st. Rspr.).

[7] 2. a) Die Verfassungsbeschwerde ist, jedenfalls soweit die angegriffenen Maßnahmen den Bf. selbst betreffen, zumindest nicht von vornherein unzulässig oder offensichtlich unbegründet. Sie bedarf eingehenderer Prüfung, was im Rahmen eines Eilverfahrens nicht möglich ist. Auch insoweit ist dem Bf. derzeit nicht entgegenzuhalten, dass er den Rechtsweg nicht erschöpft hätte. Vorherigen Rechtsschutz in der Hauptsache konnte er wegen der Kürze der Zeit nicht erlangen.

[8] b) Daher ist über den Antrag auf einstweilige Anordnung aufgrund einer Folgenabwägung $\mathrm{zu}$ entscheiden. Bei der Prüfung, ob die Voraussetzungen des $\$ 32$ Abs. 1 BVerfGG gegeben sind, ist allerdings wegen der weittragenden Folgen einer einstweiligen Anordnung grundsätzlich ein strenger Maßstab anzulegen (vgl. BVerfGE 55, 1, 3; 82, 310, 312; 94, 166, 216f.; 106, 51, 58; BVerfG, Beschl. v. 8. 6.2018 - 2 BvR 1094/18 -, Beschl. v. 1. 10.2018 - 2 BvR 1845/18 -, Rdnr. 18; Beschl. v. 23.3.2020 - 2 BvQ 6/20 -, Rdnr. 18; st. Rspr.). Bei der Folgenabwägung sind die Auswirkungen auf alle von den angegriffenen Regelungen Betroffenen zu berücksichtigen, nicht nur die Folgen für den Bf. (vgl. für förmliche Gesetze BVerfGE 122, 342, 362; 131, 47, 61).

[9] Danach ist die begehrte einstweilige Anordnung nicht zu erlassen. Der Bf. legt zwar nachvollziehbar dar, dass die angegriffenen Maßnahmen zur Bekämpfung der Corona-Pandemie seine grundrechtlich geschützten Freiheiten weitgehend verkürzen, weil er danach derzeit etwa keine Partnerschaft anbahnen, mit anderen musizieren oder demonstrieren könne. Auch ist nicht zu verkennen, dass die angegriffenen Maßnahmen zur Eindämmung der CoronaPandemie die Grundrechte der Menschen, die sich in Bayern aufhalten, erheblich beschränken. Sie geben vor, den unmittelbaren körperlichen Kontakt und weithin auch die reale Begegnung zu beschränken oder ganz zu unterlassen, sie untersagen Einrichtungen, an denen sich Menschen treffen, den Betrieb und sie verbieten es, die eigene Wohnung ohne bestimmte Gründe zu verlassen.

[10] Erginge die beantragte einstweilige Anordnung nicht und hätte die Verfassungsbeschwerde Erfolg, wären all diese Einschränkungen mit ihren erheblichen und voraussichtlich teilweise auch irreversiblen sozialen, kulturellen und wirtschaftlichen Folgen zu Unrecht verfügt und etwaige Verstöße gegen sie auch zu Unrecht geahndet worden. Erginge demgegenüber die beantragte einstweilige Anordnung und hätte die Verfassungsbeschwerde keinen Erfolg, würden sich voraussichtlich sehr viele Menschen so verhalten, wie es mit den angegriffenen Regelungen unterbunden werden soll, obwohl diese Verhaltensbeschränkungen mit der Verfassung vereinbar wären. So dürften dann insbesondere Einrichtungen, deren wirtschaftliche Existenz durch die Schließungen beeinträchtigt wird, wieder öffnen, viele Menschen ihre Wohnung häufiger verlassen und auch der unmittelbare Kontakt zwischen Menschen häufiger stattfinden. Damit würde sich die Gefahr der Ansteckung mit dem Virus, der Erkrankung vieler Personen, der Überlastung der gesundheitlichen Einrichtungen bei der Behandlung schwerwiegender Fälle und schlimmstenfalls des Todes von Menschen nach derzeitigen Erkenntnissen (ausführlich dazu BayVerfGH, Entscheidung v. 26.3.2020 - 6-VII-20 -, Rdnrn. 16f.) erheblich erhöhen.

[11] Aus der Verfassungsbeschwerde ist damit insgesamt nicht ersichtlich oder sonst erkennbar, dass die Folgen einer Fortgeltung der angegriffenen Schutzmaßnahmen gegen die Corona-Pandemie in einem Maße untragbar wären, dass ausnahmsweise eine geltende Regelung im Eilrechtsschutz außer Vollzug gesetzt werden müsste. Die hier geltend gemachten Interessen sind gewichtig, erscheinen aber nach dem hier anzulegenden strengen Maßstab nicht derart schwerwiegend, dass es unzumutbar erschiene, sie einstweilen zurückzustellen, um einen möglichst weitgehenden Gesundheits- und Lebensschutz zu ermöglichen, zu dem der Staat aus dem Grundrecht auf Leben und körperliche Unversehrtheit in Art. 2 Abs. 2 GG prinzipiell auch verpflichtet ist (vgl. BVerfGE 77, 170, 214; 85, 191, 212; 115, 25, 44f.). Gegenüber den Gefahren für Leib und Leben wiegen die Einschränkungen der persönlichen Freiheit weniger schwer. Insoweit ist auch zu berücksichtigen, dass die angegriffenen Regelungen von vornherein befristet sind, im Hinblick auf die Ausgangsbeschränkungen zahlreiche Ausnahmen vorsehen und bei der Ahndung von Verstößen im Einzelfall im Rahmen des Ermessens individuellen Belangen von besonderem Gewicht Rechnung zu tragen ist.

\section{Entstehungszeitpunkt vertragsärztlicher Honoraransprüche}

InsO §§35 Abs. 2, 55 Abs. 1, 115, 116; SGB V §85 Abs. 4

1. Ein Zahlungsdiensterahmenvertrag (Girovertrag) stellt einen Geschäftsbesorgungsvertrag dar, der durch die Eröffnung des Insolvenzverfahrens erlischt.

2. Die Freigabe von Vermögen aus der selbstständigen Tätigkeit erfasst Forderungen, die vor Wirksamwerden der Freigabeerklärung entstanden sind, auch dann nicht, wenn sie auf die bisherige selbstständige Tätigkeit des Schuldners zurückgehen.

3. Eine Honorarforderung eines Zahnarztes gegen einen Privatpatienten gehört zum Vermögen des Schuldners, sobald die Leistung erbracht ist und ein Gebührentatbestand erfüllt ist.

4. Eine Honorarforderung eines Vertragszahnarztes gegen die Kassenzahnärztliche Vereinigung gehört mit Abschluss des Quartals, in dem der Vertragszahnarzt vertragszahnärztliche Leistungen erbracht hat, und der Vorlage der entsprechenden Abrechnung bei der Kassenzahnärztlichen Vereinigung zum Vermögen des Schuldners. Für die Zuordnung von Abschlagszahlungen der Kassenzahnärztlichen Vereinigung kommt es auf den Zeitpunkt ihrer Zahlung an (im Anschluss an BSGE 118, 30; teilweise Aufgabe von BGHZ 167, $363=$ MedR 2007, 44).

BGH, Urt. v. 21. 2.2019 - IX ZR 246/17 (OLG Koblenz)

Problemstellung: In der Entscheidung des BGH geht es unter anderem um die bislang umstrittene Frage des Entstehungszeitpunkts vertragsärztlicher Honoraransprüche. Dies ist etwa von Bedeutung, wenn geklärt werden muss, ob solche Ansprüche bei Freigabe der Praxis durch den Insolvenzverwalter gemäß $₫ 35$ Abs. 2 S. 1 InsO zur Insolvenzmasse gehören. Da die Freigabe ex nunc wirkt (Kübler/Prütting/Bork/Holzer, Insolvenz-

Eingesandt und bearbeitet von Dr. iur. Niclas Lauf, Institut für Medizinrecht, Universität zu Köln, Albertus-Magnus-Platz, 50923 Köln, Deutschland 